\title{
Convergence of a Shock-Capturing Streamline Diffusion Finite Element Method for a Scalar Conservation Law in Two Space Dimensions
}

\author{
By Anders Szepessy
}

\begin{abstract}
We prove a convergence result for a shock-capturing streamline diffusion finite element method applied to a time-dependent scalar nonlinear hyperbolic conservation law in two space dimensions. The proof is based on a uniqueness result for measure-valued solutions by DiPerna. We also prove an almost optimal error estimate for a linearized conservation law having a smooth exact solution.
\end{abstract}

1. Introduction. In this note we continue the analysis of shock-capturing streamline diffusion finite element methods (SC-metods for short below) for hyperbolic conservation laws initiated by Johnson and Szepessy [6], [7], where convergence for Burgers' equation in one dimension was proved using the theory of compensated compactness. We prove here strong convergence in $L_{1}^{\text {loc }}$ for a SCmethod with piecewise linear elements applied to the following scalar conservation law in two dimensions:

$$
\begin{gathered}
u_{t}+\sum_{i=1}^{2} \frac{\partial}{\partial x_{i}} f_{i}(u)=0 \quad \text { in } R^{2} \times R_{+} \equiv R_{+}^{3}, \\
u(x, 0)=u_{0}(x) \quad \text { for } x \in R^{2},
\end{gathered}
$$

where the $f_{i}: R \rightarrow R$ are given smooth functions and we assume that the initial data $u_{0} \in L_{\infty}\left(R^{2}\right)$ have compact support. The convergence result is obtained using a uniqueness result by DiPerna [1] for measure-valued solutions by proving that the finite element solutions are uniformly bounded in $L_{\infty}$, weakly consistent with all entropy inequalities and strongly consistent with the initial condition. We also show that the accuracy of the method when applied to a linearized conservation law is at least $\mathscr{O}\left(h^{3 / 2}\right)$, where $h$ is the mesh parameter.

The streamline diffusion method is a general finite element method for hyperbolic problems which may be viewed as a certain combination of the standard Galerkin method and a least squares method. In the shock-capturing streamline diffusion method an artificial viscosity is added, with viscosity depending on the residual of the finite element solution and a certain mesh-dependent parameter (here the residual means the result of the hyperbolic operator applied to the finite element solution). The shock-capturing streamline diffusion method combines $\mathscr{O}\left(h^{3 / 2}\right)$ accuracy (in the case of piecewise linear elements) with good stability obtained through the least squares control of the residual and the shock-capturing artificial viscosity.

Received June 1, 1988.

1980 Mathematics Subject Classification (1985 Revision). Primary 65M15, 65M60. 
For more information on streamline diffusion finite element methods we refer to Hughes and Mallet [2], Johnson et al. [4] and the references therein. An extension of the convergence result of this paper to include also boundary conditions is given in [9].

The shock-capturing artificial viscosity coefficients in this paper differ from those in Hughes and Mallet [2] and Johnson et al. [7], where the artificial viscosity was normalized by dividing by the gradient of the approximate solution. We compensate by using here a smaller mesh-dependent parameter in the coefficient of the artificial viscosity. This makes no essential difference in the convergence proof for nonsmooth solutions, but makes it possible to easily prove that the error for a linearized version of $(1.1)$ is of the desired order $\mathscr{O}\left(h^{k+1 / 2}\right)$ in regions where the exact solution is smooth, when using elements of order $k$.

An outline of the paper is as follows. In Section 2 we give some background on Young measures and state the uniqueness result for measure-valued solutions satisfying entropy conditions. In Section 3 we introduce the SC-method and in the main Theorem 3.1 we prove that the finite element solutions $U^{h}$ converge strongly in $L_{p}^{\text {loc }}, 1 \leq p<\infty$, to the unique $L_{\infty}$-solution $u$ of $(1.1)$ as the mesh parameter $h$ tends to zero. The proof is divided into Lemmas 3.1-3.3, which are proved in Sections 4-6. In Lemma 3.1 we prove that $\left\|U^{h}\right\|_{L_{\infty}}$ is uniformly bounded in $h$ by proving $L_{p}$-estimates and letting $p$ tend to infinity as in [7] in the case of one space dimension. In Lemma 3.2 we prove that the Young measure associated with $U^{h}$ is a weak solution and satisfies all entropy inequalities corresponding to convex entropies. Finally, in Lemma 3.3 we prove convergence towards initial data as $h$ tends to zero by combining weak convergence and $L_{2}$-stability. In Section 7 we prove error estimates for the SC-method applied to a linearized version of (1.1) with smooth solution, thus demonstrating that the shock-capturing modification in the SC-method does not degrade the accuracy for smooth solutions. We shall denote by $C$ a positive constant not necessarily the same at each occurrence and always independent of $h$.

2. Measure-Valued Solutions. In this section we give the necessary background material on Young measures and measure-valued solutions of conservation laws following [10], [11], [1]. Our convergence result is based on Theorem 2.2 below.

THEOREM 2.1. Let $u_{j}$ be a uniformly bounded sequence in $L_{\infty}\left(R_{+}^{3}\right)$, i.e., for some constant $K$,

$$
\left\|u_{j}\right\|_{L_{\infty}\left(R_{+}^{3}\right)} \leq K, \quad j=1,2,3, \ldots
$$

Then there exists a subsequence (again denoted) $u_{j}$ and a family of measurable probability measures $\nu_{y} \in \operatorname{Prob}(R), y \in R_{+}^{3}$, such that $\operatorname{supp} \nu_{y}$ is contained in $\{x \in R:|x| \leq K\}$ and the $L_{\infty}$ weak-star limit,

$$
g\left(u_{j}(\cdot)\right) \rightarrow \bar{g}(\cdot)
$$

exists for all continuous functions $g$, where

$$
\bar{g}(y)=\int_{R} g(\lambda) d \nu_{y} \equiv\left\langle\nu_{y}, g(\lambda)\right\rangle
$$


for almost all points $y \in R_{+}^{3}$. Here, $\nu_{y}$ being measurable means that $\left\langle\nu_{y}, g\right\rangle$ is measurable with respect to $y$ for each continuous function $g$.

COROLLARY 2.1. The sequence $u_{j}$ in Theorem 2.1 converges strongly to $u$ if and only if the associated Young measure $\nu_{y}$ reduces at almost all points $y$ to a Dirac measure concentrated at $u(y)$, i.e.,

$$
\nu_{y}=\delta_{u(y)}
$$

Since $u_{j}$ is uniformly bounded in $L_{\infty}\left(R_{+}^{3}\right)$, strong convergence in $L_{p}^{\text {loc }}$ for some $p$, $1 \leq p<\infty$, is equivalent to strong convergence in $L_{q}^{\text {loc }}$ for all $q, 1 \leq q<\infty$.

We can now define the measure-valued (mv) solution of (1.1) introduced by DiPerna [1].

Definition. A measurable map $\nu: y \rightarrow \nu_{y} \in \operatorname{Prob}(R)$ from the domain $R_{+}^{3}$ is a mv solution of (1.1a) if

$$
\frac{\partial}{\partial t}\left\langle\nu_{y}, \lambda\right\rangle+\sum_{i=1}^{2} \frac{\partial}{\partial x_{i}}\left\langle\nu_{y}, f_{i}(\lambda)\right\rangle=0 \quad \text { in } \mathscr{D}^{\prime}\left(R_{+}^{3}\right)
$$

i.e., in the sense of distributions on $R_{+}^{3}$,

$$
\int_{R_{+}^{3}}\left(\phi_{t}\left\langle\nu_{y}, \lambda\right\rangle+\sum_{i=1}^{2}\left\langle\nu_{y}, f_{i}(\lambda)\right\rangle \phi_{x_{i}}\right) d x d t=0
$$

for all $\phi \in \mathscr{C}_{0}^{1}\left(R_{+}^{3}\right)$. Further, a mv solution $\nu$ of $(1.1 \mathrm{a})$ is admissible if

$$
\frac{\partial}{\partial t}\left\langle\nu_{y}, \eta(\lambda)\right\rangle+\sum_{i=1}^{2} \frac{\partial}{\partial x_{i}}\left\langle\nu_{y}, q_{i}(\lambda)\right\rangle \leq 0 \quad \text { in } \mathscr{D}^{\prime}\left(R_{+}^{3}\right),
$$

for all convex entropy pairs $(\eta, q)=\left(\eta, q_{1}, q_{2}\right)$.

We recall that $(\eta, q)$ is a convex entropy pair if the entropy $\eta: R \rightarrow R$ is continuous and convex, the corresponding entropy flux $q=\left(q_{1}, q_{2}\right): R \rightarrow R^{2}$ is continuous and all $\mathscr{C}^{1}$-solutions $u(x, t)$ of (1.1a) satisfy the additional conservation law

$$
\frac{\partial}{\partial t} \eta(u)+\sum_{i=1}^{2} \frac{\partial}{\partial x_{i}} q_{i}(u)=0 \quad \text { in } \mathscr{D}^{\prime}\left(R_{+}^{3}\right) .
$$

For smooth $\eta,(2.6)$ holds if and only if

$$
\eta^{\prime} f_{i}^{\prime}=q_{i}^{\prime}, \quad i=1,2,
$$

which means that every convex smooth function $\eta(u)$ forms an entropy pair $(\eta, q)$ provided that the corresponding entropy flux $q=\left(q_{1}, q_{2}\right)$ is defined by

$$
q_{i}(u)=\int_{0}^{u} \eta^{\prime}(s) f_{i}^{\prime}(s) d s, \quad i=1,2 .
$$

We recall that the classical admissibility condition for a solution $u$ to (1.1a) reads

$$
\frac{\partial}{\partial t} \eta(u)+\sum_{i=1}^{2} \frac{\partial}{\partial x_{i}} q_{i}(u) \leq 0 \quad \text { in } \mathscr{D}^{\prime}\left(R_{+}^{3}\right),
$$

for all convex entropy pairs. The basic existence and uniqueness result for the Kružkov $L_{\infty}$-solution $u$ to the scalar conservation law (1.1), see [8], reads as follows: 
If $u_{0} \in L_{1}\left(R^{2}\right) \cap L_{\infty}\left(R^{2}\right)$, then there exists a unique function $u \in L_{\infty}\left(R_{+}^{3}\right)$ which satisfies (2.7) for all entropies $\eta_{k}$ of the form $\eta_{k}(\lambda)=|\lambda-k|, k \in R$, together with the initial condition

$$
\lim _{t \rightarrow 0}\left\|u(\cdot, t)-u_{0}\right\|_{L_{1}\left(R^{2}\right)}=0 .
$$

The following result [1, Theorem 4.2 and Remark 3] gives an extension of the Kružkov uniqueness result to measure-valued solutions.

THEOREM 2.2. Suppose that $u_{0} \in L_{1}\left(R^{2}\right) \cap L_{\infty}\left(R^{2}\right)$ and that $\nu$ is an admissible $m v$ solution of (1.1a) generated by a uniformly bounded sequence $u_{j}$ in $L_{\infty}\left(R_{+}^{3}\right)$, such that for some constant $C$

$$
\begin{gathered}
\int_{R^{2}}\left\langle\nu_{y},|\lambda|\right\rangle d x \leq C, \\
\lim _{t \rightarrow 0^{+}} \frac{1}{t} \int_{0}^{t} \int_{R^{2}}\left\langle\nu_{y},\left|\lambda-u_{0}(x)\right|\right\rangle d x d t=0 .
\end{gathered}
$$

Then $\nu$ is the Dirac solution $\nu_{y}=\delta_{u(y)}$, where $u$ is the unique $L_{\infty}$-solution of (1.1).

According to Corollary 2.1 we then have $u_{j} \rightarrow u$ strongly in $L_{p}^{\text {loc }}, 1 \leq p<\infty$.

3. Formulation of the Method and the Main Theorem. In this section we formulate the SC-method and give a basic $L_{2}$-stability result and some interpolation estimates, which will be used below.

The SC-method is based on a space-time finite element discretization of $R_{+}^{3}$ defined as follows. Let $0=t_{0}<t_{1}<t_{2}<\cdots<t_{\tilde{N}}=T$ be a sequence of time levels with $t_{n+1}-t_{n} \sim h$, set $I_{n}=\left(t_{n}, t_{n+1}\right)$ and introduce the "slabs" $S_{n}=R^{2} \times I_{n}$ and the sets $R_{n}^{2}=R^{2} \times\left\{t_{n}\right\}$. Let $\tilde{T}_{h}^{n}$ be a quasi-uniform triangulation of $R^{2}$ into triangles $\tilde{K}$ with one right angle (cf. Remark 3.1), smallest angle uniformly bounded away from zero and diameter $h_{K} \sim h$. For each $\tilde{K} \in \tilde{T}_{h}^{n}$, let the prism $\tilde{K} \times I_{n}$ be divided into three tetrahedrons

$$
T(\tilde{K}, 1)=f e a b, \quad T(\tilde{K}, 2)=\operatorname{decb}, \quad T(\tilde{K}, 3)=a b c e,
$$

according to Figure 3.1, and form the corresponding "triangulation" $T_{h}^{n}=\{T(\tilde{K}, i)$, $\left.i=1,2,3, \forall \tilde{K} \in \tilde{T}_{h}^{n}\right\}$ of $S_{n}$ into tetrahedrons.

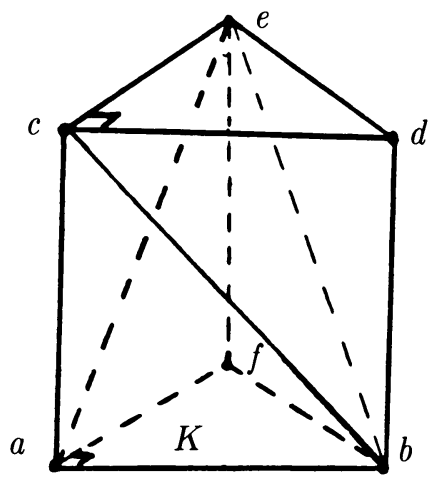

FIGURE 3.1 
Remark 3.1. The requirement that the triangles $\tilde{K} \in \tilde{T}_{h}^{n}$ have one right angle is technically convenient when proving Lemma 4.2 and Proposition 5.3. We expect these results to hold also for a more general triangulation of $R^{2} \times R_{+}$. In [9] we prove the corresponding results for a general triangulation of $R \times R_{+}$.

Define

$$
\begin{aligned}
V_{h}^{n} & =\left\{v \in H^{1}\left(S_{n}\right):\left.v\right|_{K} \in P_{1}(K) \forall K \in T_{h}^{n},\left.v\right|_{R_{+}^{3} \backslash \Omega_{\tilde{R}+2, M}}=0\right\}, \\
\Omega_{s, s^{\prime}} & =\left\{(x, t) \in R_{+}^{3}:|x|<s+t s^{\prime}\right\}, \quad s, s^{\prime}>0, \\
T_{h} & =\bigcup_{n \geq 0} T_{h}^{n},
\end{aligned}
$$

where $P_{1}(K)$ denotes the set of linear functions on $K, \tilde{R}=\max \left\{|x| ; x \in \operatorname{supp} u_{0}\right\}$ and $M$ is a positive constant to be defined below. In other words, $V_{h}^{n}$ consists of continuous piecewise linear functions on the slab $S_{n}$ which are zero for large $|x|$.

We shall seek an approximate solution $U^{h}$ in the space $V_{h} \equiv \prod_{n=0}^{N} V_{h}^{n}$, i.e., for $n=0,1,2, \ldots, N$ we will have $\left.U^{h}\right|_{S_{n}} \in V_{h}^{n}$. Note that the functions in $V_{h}$ are continuous in $x$ and possibly discontinuous in $t$ at the discrete time levels $t_{n}$. The SC-method for (1.1) can now be formulated: Find $U^{h} \in V_{h}$ such that for $n=0,1,2, \ldots$

$$
\begin{array}{rl}
\int_{S_{n}} & L\left(U^{h}\right)\left(v+\delta\left(v_{t}+\sum_{i=1}^{2} f_{i}^{\prime}\left(U^{h}\right) v_{x_{i}}\right)\right) d x d t+\int_{R_{n}^{2}}\left(U_{+}^{h}-U_{-}^{h}\right) v_{+} d x \\
& +\int_{S_{n}} \varepsilon_{1}\left(U^{h}\right) \nabla U^{h} \cdot \nabla v d x d t \\
& +\int_{S_{n}} \varepsilon_{2}\left(U^{h}\right) \nabla_{x} U^{h} \cdot \nabla_{x} v d x d t=0 \quad \forall v \in V_{h}
\end{array}
$$

where

$$
\begin{aligned}
& L\left(U^{h}\right)=U_{t}^{h}+\sum_{i=1}^{2}\left(f_{i}\left(U^{h}\right)\right)_{x_{i}} \\
& \varepsilon_{1}\left(U^{h}\right)=\bar{\delta}\left|L\left(U^{h}\right)\right|\left(1+\left|f_{1}^{\prime}\left(U^{h}\right)\right|+\left|f_{2}^{\prime}\left(U^{h}\right)\right|\right), \\
& \varepsilon_{2}\left(U^{h}\right)=\overline{\bar{\delta}}|\tilde{U}| \text {, } \\
& v_{ \pm}(\cdot, t)=\lim _{s \rightarrow 0^{ \pm}} v(\cdot, t+s) \text {, } \\
& \left.\tilde{U}(x, t)\right|_{K}= \begin{cases}\left.\left(U_{+}^{h}-U_{-}^{h}\right)(x)\right|_{K \cap R_{n}^{2}} & \text { if } \int_{\bar{K} \cap R_{n}^{2}} d x>0 \text { for } K \in T_{h}^{n}, \\
0 & \text { otherwise, }\end{cases} \\
& U_{-}(\cdot, 0)=u_{0}(\cdot) \text {, } \\
& \nabla_{x} w \cdot \nabla_{x} v=\sum_{i=1}^{2} v_{x_{i}} w_{x_{i}} \\
& \nabla w \cdot \nabla v=w_{t} v_{t}+\nabla_{x} w \cdot \nabla_{x} v
\end{aligned}
$$

and the positive parameters $\delta, \bar{\delta}, \overline{\bar{\delta}}$ satisfy

$$
h / \delta \rightarrow 0 \text { and } \delta / h^{1-\varepsilon} \rightarrow 0 \text { as } h \rightarrow 0, \forall \varepsilon>0 \text {, }
$$$$
\bar{\delta}=C h^{\alpha_{1}}, \quad \overline{\bar{\delta}}=C h^{\alpha_{2}},
$$

where $\frac{3}{2}<\alpha_{1}<2, \frac{1}{2}<\alpha_{2}<1$. 
Existence of a solution to (3.1) follows from a variant of Brouwer's fixed point theorem as in [5]. From now on, $U=U^{h}$ will denote a solution of (3.1). Our main result is the following

THEOREM 3.1. The solutions $U^{h}$ of $(3.1)$ converge strongly in $L_{p}^{\text {loc }}\left(R_{+}^{3}\right)$ for $1 \leq p<\infty$ to the unique $L_{\infty}$-solution of (1.1) as $h$ tends to zero.

The proof is divided into three steps: Lemmas 3.1-3.3.

LEMMA 3.1. There is a constant $C$ such that the solutions $U^{h}$ of (3.1) satisfy

$$
\left\|U^{h}\right\|_{L_{\infty}\left(R_{+}^{3}\right)} \leq C, \quad 0<h<1 .
$$

LEMMA 3.2. There is a subsequence of the solutions $U^{h}$ of (3.1) that generates an admissible $m v$ solution $\nu$ of (1.1).

LEMMA 3.3. There is a constant $C$ such that the $m v$ solution $\nu$ in Lemma 3.2 satisfies

and the initial condition

$$
\int_{R^{2}}\left\langle\nu_{y},|\lambda|\right\rangle d x \leq C
$$

$$
\lim _{t \rightarrow 0^{+}} \frac{1}{t} \int_{0}^{t}\left\langle\nu_{y},\left|\lambda-u_{0}(x)\right|\right\rangle d x d t=0 .
$$

Theorem 3.1 now directly follows by combining Lemmas 3.1-3.3, Theorem 2.2 and Corollary 2.1.

Next, we give the following basic $L_{2}$-stability result obtained by taking $v=U^{h}$ in $(3.1)$ :

$$
\begin{aligned}
\int_{R_{N+1}^{2}} & \left(U_{-}^{h}\right)^{2} d x+\sum_{n=0}^{N} \int_{R_{n}^{2}}\left(U_{+}^{h}-U_{-}^{h}\right)^{2} d x+2 \delta \int_{S^{N}}\left(L\left(U^{h}\right)\right)^{2} d x d t \\
& +2 \int_{S^{N}} \varepsilon_{1}\left(U^{h}\right)\left|\nabla U^{h}\right|^{2} d x d t+2 \int_{S^{N}} \varepsilon_{2}\left(U^{h}\right)\left|\nabla_{x} U^{h}\right|^{2} d x d t \\
& \leq \int_{R^{2}}\left(u_{0}\right)^{2} d x
\end{aligned}
$$

where $S^{N}=\bigcup_{n=0}^{N} S_{n}$ and integrals over $S^{N}$ are interpreted as a sum of integrals over the $S_{n}$.

We shall need the following standard interpolation error estimate (3.4), "superapproximation" result (3.5) and inverse estimate (3.6), where $\pi w \in V_{h}$ is the usual piecewise linear interpolant of a function $w \in \Pi_{n \geq 0} \mathscr{C}\left(S_{n}\right)$. A proof of the superapproximation result is given in [7].

LEMMA 3.4. There are constants $C$ such that for $w \in W^{s, p}(\omega) \cap \mathscr{C}\left(S_{n}\right), v \in V_{h}$, $n=0,1,2, \ldots$,

$$
\begin{array}{cl}
\|w-\pi w\|_{W^{k, \infty}(\omega)} \leq C h^{s-k}\|w\|_{\dot{W}^{s, \infty}(\omega)}, & s=1,2, k=0,1, p=\infty \\
\|w-\pi w\|_{H^{k}(\omega)} \leq C h^{2-k}\|w\|_{\dot{H}^{2}(\omega)}, & k=0,1, p=2 \\
\|v w-\pi(v w)\|_{W^{k, \infty}(\omega)} & \\
\leq C h^{1-k}\|v\|_{L_{\infty}(\omega)}\|w\|_{\dot{W}^{1, \infty}(\omega)}, & k=0,1, p=\infty
\end{array}
$$




$$
\begin{aligned}
& \|v w-\pi(v w)\|_{H^{k}(\omega)} \\
& \leq C h^{1-k}\|v\|_{L_{\infty}(\omega)}\left(\|w\|_{H^{1}(\omega)}+h\|w\|_{\dot{H}^{2}(\omega)}\right), \quad k=0,1, p=2, \\
& \|v w-\pi(v w)\|_{L_{2}\left(R_{n}^{2}\right)} \\
& \leq C h^{1 / 2}\|v\|_{L_{\infty}\left(S_{n}\right)}\left(\|w\|_{H^{1}\left(S_{n}\right)}+h\|w\|_{\dot{H}^{2}\left(S_{n}\right)}\right), \quad p=2 \\
& \|\nabla v\|_{L_{p}(\omega)} \leq C h^{-1}\|v\|_{L_{p}(\omega)}, \quad 1 \leq p \leq \infty \\
& \|v\|_{L_{\infty}\left(S_{n}\right)} \leq C h^{-3 / 2}\|v\|_{L_{2}\left(S_{n}\right)}, \quad p=2
\end{aligned}
$$

where $\omega=R_{n}^{2}, S_{n}, K \cap R_{n}^{2}$ or $K \cap S_{n}$ for $K \in T_{h}$, and $W^{s, p}(\omega)$ is the usual Sobolev space (here dot denotes seminorm and $W^{s, 2}=H^{2}$ ).

4. Proof of Lemma 3.1 (The $L_{\infty}$-Estimate). In order to prove the $L_{\infty}$ estimate of Lemma 3.1, we shall need the following two preliminary lemmas.

LEMMA 4.1. There are positive constants $c$ and $C$ independent of $p$ such that for $p=2 m, m=1,2,3, \ldots$, and $n=0,1,2, \ldots$

$$
\begin{aligned}
& \frac{c h}{p^{2}} \sum_{K \in T_{h}^{n}} \int_{K \cap R_{n}^{2}}\left|U_{+}-U_{-}\right|\left|\nabla_{x} U_{+}\right|^{2}\left\|U_{+}\right\|_{L_{\infty}\left(K \cap R_{n}^{2}\right)}^{p-2} d x \\
& \quad \leq \int_{S_{n}}|\tilde{U}| \nabla_{x} U \cdot \nabla_{x}\left(\pi\left(U^{p-1}\right)\right) d x d t \\
& \operatorname{ch} \int_{R_{n}^{2}}\left|U_{+}-U_{-}\right| \nabla_{x} U_{+} \cdot \nabla_{x} v_{+} d x \leq \int_{S_{n}}|\tilde{U}|\left|U_{x} U \cdot \nabla_{x} v\right| d x d t \\
& \quad \leq C h \int_{R_{n}^{2}}\left|U_{+}-U_{-}\right|\left|\nabla_{x} U_{+} \cdot \nabla_{x} v_{+}\right| d x \quad \forall v \in V_{h} .
\end{aligned}
$$

The proof of Lemma 4.1 is a simple combination of the proofs of Lemmas 4.1-4.2 in [7].

LEMMA 4.2. There is a constant $c>0$ independent of $p$ such that for $p=2 m$, $m=1,2,3, \ldots$, and $n=0,1,2, \ldots$

$$
\int_{S_{n}} \varepsilon_{1}(U) \nabla U \cdot \nabla \pi\left(U^{p-1}\right) d x d t \geq \frac{c}{p^{2}} \sum_{K \in T_{h}^{n}} \int_{K} \varepsilon_{1}(U)|\nabla U|^{2}\|U\|_{L_{\infty}(K)}^{p-2} d x d t .
$$

Proof. Consider a tetrahedron $K \in T_{h}^{n}$. Since

$$
\left.\nabla U \cdot \nabla \pi\left(U^{p-1}\right)\right|_{K} \quad \text { and } \quad|\nabla U|^{2}\|U\|_{L_{\infty}(K)}^{p-2}
$$

are constant on $K$, it is sufficient to prove that

$$
\nabla U \cdot \nabla \pi\left(U^{p-1}\right) \geq \frac{c}{p^{2}}|\nabla U|^{2}\|U\|_{L_{\infty}(K)}^{p-2} .
$$

Define the function $f_{p}: S^{3} \backslash\left\{ \pm \frac{1}{2}(1,1,1,1)\right\} \rightarrow R$ by

$f_{p}\left(y_{1}, y_{2}, y_{3}, y_{4}\right)$

$$
\begin{aligned}
& =\frac{\left(y_{1}-y_{2}\right)\left(y_{1}^{p-1}-y_{2}^{p-1}\right)+\left(y_{1}-y_{3}\right)\left(y_{1}^{p-1}-y_{3}^{p-1}\right)+\left(y_{3}-y_{4}\right)\left(y_{3}^{p-1}-y_{4}^{p-1}\right)}{\left(\left(y_{1}-y_{2}\right)^{2}+\left(y_{1}-y_{3}\right)^{2}+\left(y_{3}-y_{4}\right)^{2}\right) \max \left(y_{1}^{p-2}, y_{2}^{p-2}, y_{3}^{p-2}, y_{4}^{p-2}\right)} \\
& =\frac{\left(y_{1}-y_{2}\right)^{2} \sum_{i=0}^{p-2} y_{2}^{p-2-i} y_{1}^{i}+\left(y_{1}-y_{3}\right)^{2} \sum_{i=0}^{p-2} y_{3}^{p-2-i} y_{1}^{i}+\left(y_{3}-y_{4}\right)^{2} \sum_{i=0}^{p-2} y_{4}^{p-2-i} y_{3}^{i}}{\left(\left(y_{1}-y_{2}\right)^{2}+\left(y_{1}-y_{3}\right)^{2}+\left(y_{3}-y_{4}\right)^{2}\right) \max \left(y_{1}^{p-2}, y_{2}^{p-2}, y_{3}^{p-2}, y_{4}^{p-2}\right)} .
\end{aligned}
$$


We note that $f_{p}$ is continuous on $S^{3}$, and after an appropriate choice of coordinate directions we get by the definition of $f_{p}$

$$
\nabla U \cdot \nabla \pi\left(U^{p-1}\right) \geq \inf _{y \in S^{3}} f_{p}(y)|\nabla U|^{2}\|U\|_{L_{\infty}(K)}^{p-2} .
$$

We have

$$
f_{p}(y) \geq \frac{1}{4} \frac{\left(y_{1}-y_{2}\right)^{2}\left(y_{1}^{p-2}+y_{2}^{p-2}\right)+\left(y_{1}-y_{3}\right)^{2}\left(y_{1}^{p-2}+y_{3}^{p-2}\right)+\left(y_{3}-y_{4}\right)^{2}\left(y_{3}^{p-2}+y_{4}^{p-2}\right)}{\left(\left(y_{1}-y_{2}\right)^{2}+\left(y_{1}-y_{3}\right)^{2}+\left(y_{3}-y_{4}\right)^{2}\right) \max \left(y_{1}^{p-2}, y_{2}^{p-2}, y_{3}^{p-2}, y_{4}^{p-2}\right)} .
$$

Let us first assume that $\left|y_{1}\right|^{p-2}=\max _{1 \leq i \leq 4}\left(y_{i}^{p-2}\right)$. If $\left(y_{1}-y_{2}\right)^{2}+\left(y_{1}-y_{3}\right)^{2} \geq 1 / p^{2}$, then we clearly have

$$
f_{p}(y)>c / p^{2}
$$

In the case $\left(y_{1}-y_{2}\right)^{2}+\left(y_{1}-y_{3}\right)^{2}<1 / p^{2}$, we have

$$
\left(\frac{y_{i}}{y_{1}}\right)^{p-2}=\left(1-\frac{y_{1}-y_{i}}{y_{1}}\right)^{p-2}>\exp \left(-\frac{2(p-2)}{p\left|y_{1}\right|}\right)>c, \quad i=2,3
$$

so that

$$
\begin{aligned}
f_{p}(y) \geq & \frac{1}{4}\left(\frac{y_{2}}{y_{1}}\right)^{p-2} \frac{\left(y_{1}-y_{2}\right)^{2}}{\left(y_{1}-y_{2}\right)^{2}+\left(y_{1}-y_{3}\right)^{2}+\left(y_{3}-y_{4}\right)^{2}} \\
& +\frac{1}{4}\left(\frac{y_{3}}{y_{1}}\right)^{p-2} \frac{\left(y_{1}-y_{3}\right)^{2}+\left(y_{3}-y_{4}\right)^{2}}{\left(y_{1}-y_{2}\right)^{2}+\left(y_{1}-y_{3}\right)^{2}+\left(y_{3}-y_{4}\right)^{2}}>c
\end{aligned}
$$

which proves (4.1).

Next, we consider the case $\left|y_{2}\right|^{p-2}=\max _{1 \leq i \leq 4}\left(\left|y_{i}\right|^{p-2}\right)$. If $\left|y_{1}-y_{2}\right| \geq 1 / p$, then (4.1) follows directly, while if $\left|y_{1}-y_{2}\right|<1 / p$, then we have as in (4.2)

$$
\begin{aligned}
f_{p}(y) & \geq \frac{1}{4} \frac{\left(y_{1} / y_{2}\right)^{p-2}\left(\left(y_{1}-y_{2}\right)^{2}+\left(y_{1}-y_{3}\right)^{2}\right)+\left(y_{3} / y_{2}\right)^{p-2}\left(y_{3}-y_{4}\right)^{2}}{\left(y_{1}-y_{2}\right)^{2}+\left(y_{1}-y_{3}\right)^{2}+\left(y_{3}-y_{4}\right)^{2}} \\
& \geq \frac{1}{4} \frac{c\left(\left(y_{1}-y_{2}\right)^{2}+\left(y_{1}-y_{3}\right)^{2}\right)+\left(y_{3} / y_{2}\right)^{p-2}\left(y_{3}-y_{4}\right)^{2}}{\left(y_{1}-y_{2}\right)^{2}+\left(y_{1}-y_{3}\right)^{2}+\left(y_{3}-y_{4}\right)^{2}} .
\end{aligned}
$$

If now $\left|y_{1}-y_{3}\right| \geq 1 / p$, then (4.1) holds, and finally, if $\left|y_{1}-y_{3}\right|<1 / p$, then we have $\left|y_{3}-y_{2}\right|<\left|y_{1}-y_{2}\right|+\left|y_{1}-y_{3}\right|<2 / p$, and as in (4.2) we obtain (4.1). By symmetry, this proves (4.1) for all $y \in S^{3}$.

Taking now $v=\pi\left(U^{p-1}\right)$ in (3.1), where $p$ is an even integer greater than 2, we get

$$
\begin{aligned}
0= & \int_{S_{n}} L(U) U^{p-1} d x d t+\int_{R_{n}^{2}}\left(U_{+}-U_{-}\right)\left(U_{+}\right)^{p-1} d x \\
& -\int_{S_{n}} L(U)\left(U^{p-1}-\pi\left(U^{p-1}\right)\right) d x d t-\int_{R_{n}^{2}}\left(U_{+}-U_{-}\right)\left(U_{+}^{p-1}-\pi\left(U_{+}^{p-1}\right)\right) d x \\
& +\delta \int_{S_{n}} L(U)\left(\left(U^{p-1}\right)_{t}+\sum_{i} f_{i}^{\prime}(U)\left(U^{p-1}\right)_{x_{i}}\right) d x d t \\
& -\delta \int_{S_{n}} L(U)\left(\left(U^{p-1}\right)_{t}-\pi\left(U^{p-1}\right)_{t}+\sum_{i} f_{i}^{\prime}(U)\left(\left(U^{p-1}\right)_{x_{i}}-\pi\left(U^{p-1}\right)_{x_{i}}\right)\right) d x d t \\
& +\int_{S_{n}} \varepsilon_{1}(U) \nabla U \cdot \nabla \pi\left(U^{p-1}\right) d x d t \\
& +\int_{S_{n}} \varepsilon_{2}(U) \nabla_{x} U \cdot \nabla_{x} \pi\left(U^{p-1}\right) d x d t \equiv \sum_{i=1}^{8} E_{n}^{i} .
\end{aligned}
$$


Using the standard interpolation estimates (3.4) and the stability estimate (3.3), we have

$$
\begin{aligned}
\left|E_{n}^{3}\right|+\left|E_{n}^{6}\right| \leq & C p^{2} h(h+\delta) \sum_{K \in T_{h}^{n}} \int_{K}|L(U)|\left(1+\sum_{i}\left|f_{i}^{\prime}(U)\right|\right)|\nabla U|^{2}\|U\|_{L_{\infty}(K)}^{p-3} \\
\leq & C p^{2} \frac{h \delta}{\bar{\delta}} \sum_{K \in T_{h}^{n}} \int_{K \cap\{|U|>1\}} \varepsilon_{1}(U)|\nabla U|^{2}\|U\|_{L_{\infty}(K)}^{p-2} d x d t \\
& +C p^{2} \frac{h \delta}{\bar{\delta}} \int_{S_{n}} \varepsilon_{1}(U)|\nabla U|^{2} d x d t=\mathrm{I}_{n}+\mathrm{II}_{n}
\end{aligned}
$$

where by Lemma 4.2

$$
\left|\mathrm{I}_{n}\right| \leq \frac{C p^{4} h \delta}{\bar{\delta}} \int_{S_{n}} \varepsilon_{1}(U) \nabla U \cdot \nabla \pi\left(U^{p-1}\right) d x d t
$$

and by (3.3)

$$
\sum_{n=0}^{N}\left|\mathrm{II}_{n}\right| \leq C p^{2} h \delta / \bar{\delta}
$$

Further, using (3.3) and Lemma 4.1, we have

$$
\begin{aligned}
\left|E_{n}^{4}\right| \leq & C p^{2} h^{2} \sum_{K \in T_{h}^{n}} \int_{K \cap R_{n}^{2}}\left|U_{+}-U_{-}\right|\left|\nabla_{x} U\right|^{2}\|U\|_{L_{\infty}\left(K \cap R_{n}^{2}\right)}^{p-3} d x \\
\leq & C p^{2} h^{2} \sum_{K \in T_{h}^{n}} \int_{K \cap\{|U|>1\}}\left|U_{+}-U_{-}\right|\left|\nabla_{x} U\right|^{2}\|U\|_{L_{\infty}\left(K \cap R_{n}^{2}\right)}^{p-2} d x \\
& +C p^{2} h^{2} \int_{R_{n}^{2} \cap\{|U|<1\}}\left|U_{+}-U_{-}\right|\left|\nabla_{x} U\right|^{2} d x \\
\leq & C p^{4} h \int_{S_{n}}|\tilde{U}| \nabla_{x} U \cdot \nabla_{x} \pi\left(U^{p-1}\right) d x d t+C p^{2} h \int_{S_{n}}|\tilde{U}|\left|\nabla_{x} U\right|^{2} d x d t .
\end{aligned}
$$

With the aid of these estimates we get by summation over $n=0,1,2, \ldots, N$ for $p^{4} \leq C \min (\bar{\delta} /(h \delta), \overline{\bar{\delta}} / h)$

$$
\begin{aligned}
& \sum_{n=0}^{N} \int_{R_{n+1}^{2}}\left(U_{-}\right)^{p} d x-\int_{R_{n}^{2}}\left(U_{+}\right)^{p} d x-\int_{R_{n}^{2}}\left(U_{-}-U_{+}\right) p\left(U_{+}\right)^{p-1} d x \\
& \quad+\delta p(p-1) \int_{S^{N}}(L(U))^{2} U^{p-2} d x d t \leq C p^{3}\left(\frac{h \delta}{\bar{\delta}}+\frac{h}{\overline{\bar{\delta}}}\right) .
\end{aligned}
$$

Using now the convexity of the function $U \rightarrow U^{p}$, we have

$$
\begin{gathered}
\left\|U_{-}\right\|_{L_{p}\left(R_{N+1}^{2}\right)}^{p}+\delta p(p-1) \int_{S^{N}}(L(U))^{2} U^{p-2} d x d t \\
\leq\left\|u_{0}\right\|_{L_{p}\left(R^{2}\right)}^{p}+C p^{3}(h / \overline{\bar{\delta}}+h \delta / \bar{\delta}) .
\end{gathered}
$$


The next step is to obtain $L_{p}$-estimates for all $t \in(0, \infty)$. As in [7], we have for $t_{n}<t \leq t_{n+1}$

$$
\begin{aligned}
\| U(\cdot, t) & \left\|_{L_{p}\left(R^{2}\right)}^{p}=\right\| U_{-} \|_{L_{p}\left(R_{n+1}^{2}\right)}^{p}-p \int_{t}^{t_{n+1}} \int_{R^{2}}\left(U_{t} U^{p-1}+\sum_{i} f_{i}^{\prime} U^{p-1} U_{x_{i}}\right) d x d t \\
\leq & \left\|U_{-}\right\|_{L_{p}\left(R_{n+1}^{2}\right)}^{p}+p\left(\int_{S_{n}}(L(U))^{2} U^{p-2} d x d t \int_{t}^{t_{n+1}} \int_{R^{2}} U^{p} d x d t\right)^{1 / 2} \\
\leq & \left\|U_{-}\right\|_{L_{p}\left(R_{n+1}^{2}\right)}^{p}+\delta p(p-1) \int_{S_{n}}(L(U))^{2} U^{p-2} d x d t \\
& +\frac{p}{4 \delta(p-1)} \int_{t}^{t_{n+1}}\|U(\cdot, s)\|_{L_{p}\left(R^{2}\right)}^{p} d s .
\end{aligned}
$$

Thus, by Gronwall's inequality we obtain for $t_{N} \leq t \leq t_{N+1}$

$$
\|U(\cdot, t)\|_{L_{p}\left(R^{2}\right)}^{p} \leq e^{C h / \delta}\left\|u_{0}\right\|_{L_{p}\left(R^{2}\right)}^{p}+C p^{3}(h / \overline{\bar{\delta}}+h \delta / \bar{\delta}) .
$$

This proves by (3.2) the existence of positive constants $c$ and $\alpha_{0}$, independent of $p$ and $h$, such that

$$
\sup _{t \geq 0}\|U(\cdot, t)\|_{L_{p}\left(R^{2}\right)} \leq C \quad \text { if } 4 \leq p \leq c h^{-\alpha_{0}} .
$$

Further, there is a constant $C$, independent of $q$ and $h$, such that all $v \in V_{h}$ satisfy the inverse estimate

$$
\left\|v_{ \pm}\right\|_{L_{\infty}\left(R_{n}^{2}\right)} \leq\left(C q h^{-1}\right)^{2 / q}\left\|v_{ \pm}\right\|_{L_{q}\left(R_{n}^{2}\right)}, \quad 1 \leq q<\infty
$$

which is proved analogously to Lemma 4.1. Finally, using (4.4)-(4.5), we have

$$
\begin{aligned}
\left\|U^{h}\right\|_{L_{\infty}\left(R_{+}^{3}\right)} & =\|U\|_{L_{\infty}\left(R_{+}^{3}\right)} \leq C\left(p h^{-1}\right)^{2 / p} \sup _{t \geq 0}\|U(\cdot, t)\|_{L_{p}\left(R^{2}\right)} \\
& \leq C \exp \left(c\left(1+\alpha_{0}\right) h^{\alpha_{0}} \ln 1 / h\right) \leq C,
\end{aligned}
$$

for $h$ sufficiently small, which completes the proof of Lemma 3.1.

5. Proof of Lemma 3.2 (The Entropy Condition). To prove Lemma 3.2, we first note that by Lemma 3.1 the solutions $U^{h}$ of (3.1) are uniformly bounded in the $L_{\infty}$-norm, so that by Theorem 2.1 there exists a subsequence $\left\{U^{h}\right\}$ which converges in the weak-star topology in $L_{\infty}\left(R_{+}^{3}\right)$, and the limit can be represented by a family of probability measures $\nu_{y}$ such that for all continuous state variables $g$

$$
g\left(U^{h}(y)\right) \rightarrow\left\langle\nu_{y}, g(\lambda)\right\rangle
$$

Our next step is to prove that the Young measure $\nu$ is an admissible mv solution, i.e., that $\nu$ satisfies in the distribution sense

$$
\frac{\partial}{\partial t}\left\langle\nu_{y}, \lambda\right\rangle+\sum_{i=1}^{2} \frac{\partial}{\partial x_{i}}\left\langle\nu_{y}, f_{i}(\lambda)\right\rangle=0,
$$

and for all convex entropy pairs $(\eta, q)$

$$
\frac{\partial}{\partial t}\left\langle\nu_{y}, \eta(\lambda)\right\rangle+\sum_{i=1}^{2} \frac{\partial}{\partial x_{i}}\left\langle\nu_{y}, q_{i}(\lambda)\right\rangle \leq 0 .
$$


To prove (5.3), let $\left(\eta, q_{1}, q_{2}\right)$ be a smooth convex entropy pair. Let $\Phi \in$ $\mathscr{C}_{0}^{\infty}\left(R^{2} \times R_{+}\right), \Phi \geq 0$, and partition $\Phi$ as follows:

$$
\Phi=\phi+\varphi
$$

where $\phi, \varphi \geq 0, \phi \in \mathscr{C}_{0}^{\infty}\left(\Omega_{\tilde{R}+2, M}\right)$ and $\varphi \in \mathscr{C}_{0}^{\infty}\left(R^{2} \times R_{+} \backslash \Omega_{\tilde{R}+1, M}\right)$. Taking $v=\pi\left(\eta^{\prime}(U) \phi\right)$ in (3.1), we get

$$
\begin{aligned}
0= & \int_{S_{n}} L(U) \eta^{\prime} \phi d x d t+\int_{R_{n}^{2}}\left(U_{+}-U_{-}\right) \eta_{+}^{\prime} \phi d x \\
& -\int_{S_{n}} L(U)\left(\eta^{\prime} \phi-\pi\left(\eta^{\prime} \phi\right)\right) d x d t-\int_{R_{n}^{2}}\left(U_{+}-U_{-}\right)\left(\eta^{\prime} \phi-\pi\left(\eta^{\prime} \phi\right)\right)_{+} d x \\
& +\delta \int_{S_{n}} L(U)\left(\left(\eta^{\prime} \phi\right)_{t}+\sum_{i} f_{i}^{\prime}\left(\eta^{\prime} \phi\right)_{x_{i}}\right) d x d t \\
& -\delta \int_{S_{n}} L(U)\left(\left(\eta^{\prime} \phi\right)_{t}-\pi\left(\eta^{\prime} \phi\right)_{t}+\sum_{i} f_{i}^{\prime}\left(\eta^{\prime} \phi-\pi\left(\eta^{\prime} \phi\right)\right)_{x_{i}}\right) d x d t \\
& +\int_{S_{n}} \varepsilon_{1}(U) \nabla U \cdot \nabla \pi\left(\eta^{\prime} \phi\right) d x d t+\int_{S_{n}} \varepsilon_{2}(U) \nabla_{x} U \cdot \nabla_{x} \pi\left(\eta^{\prime} \phi\right) d x d t \equiv \sum_{i=1}^{8} E_{n}^{i} .
\end{aligned}
$$

Integrating by parts and summing over $n$, we have

$$
-\int_{S^{N}}\left(\eta(U) \phi_{t}+\sum_{i} q_{i}(U) \phi_{x_{i}}\right) d x d t
$$

$$
\begin{aligned}
& +\sum_{n=0}^{N}\left(\int_{R_{n+1}^{2}} \eta\left(U_{-}\right) \phi d x-\int_{R_{n}^{2}}\left(\eta\left(U_{+}\right)-\left(U_{+}-U_{-}\right) \eta^{\prime}\left(U_{+}\right)\right) \phi d x\right) \\
= & -\sum_{n=0}^{N} \sum_{i=3}^{8} E_{n}^{i} \equiv-\sum_{i=3}^{8} R^{i} .
\end{aligned}
$$

Using now the convexity of $\eta$, we see that the sum of the integrals over $R_{n}^{2}, R_{n+1}^{2}$ is nonnegative.

Proposition 5.1. There holds

$$
\liminf _{h \rightarrow 0} \sum_{i=3}^{8} R^{i} \geq 0
$$

Proposition 5.2. There are positive constants $c, C$ such that

$$
\|U\|_{L_{\infty}\left(R^{2} \times R_{+} \backslash \Omega_{\tilde{R}+1, M}\right)} \leq C e^{-c /(h+\delta+\overline{\bar{\delta}}+\bar{\delta} / \sqrt{\delta})},
$$

where $\tilde{R}=\left\{|x|: x \in \operatorname{supp} u_{0}\right\}$ and $M$ is a positive constant to be defined below. 
We postpone the proofs of these results to the end of this section. From (5.4) we get

$$
\begin{aligned}
-\int_{S^{N}} & \left(\eta(U) \Phi_{t}+\sum_{i} q_{i}(U) \Phi_{x_{i}}\right) d x d t \\
\leq & -\sum_{i=3}^{8} R^{i}-\int_{S^{N}}\left(\eta(U) \varphi_{t}+\sum_{i} q_{i}(U) \varphi_{x_{i}}\right) d x d t \\
= & -\sum_{i=3}^{8} R^{i}-\int_{S^{N}}\left(\eta(0) \varphi_{t}+\sum_{i} q_{i}(0) \varphi_{x_{i}}\right) d x d t \\
& +\int_{S^{N}}\left((\eta(0)-\eta(U)) \varphi_{t}+\sum_{i}\left(q_{i}(0)-q_{i}(U)\right) \varphi_{x_{i}}\right) d x d t
\end{aligned}
$$

where the first integral on the right-hand side is zero and the second tends to zero as $h \rightarrow 0$ by Proposition 5.2. Letting $h \rightarrow 0$ in (5.5), using (5.1) and Proposition 5.1 , we now obtain (5.3).

In order to treat all convex continuous entropies $\eta$, we observe that a standard regularization $\eta^{\varepsilon}=\eta * \omega_{\varepsilon}$ maintains the convexity, and $\eta^{\varepsilon}, q_{i}^{\varepsilon}$ tend to $\eta, q_{i}$ uniformly. Here, $\omega$ satisfies the following conditions:

$$
\omega \in \mathscr{C}_{0}^{\infty}((-1,1)), \quad \omega \geq 0, \quad \int_{R} \omega d y=1, \quad \omega_{\varepsilon}(y)=\varepsilon^{-1} \omega\left(\frac{y}{\varepsilon}\right) .
$$

By dominated convergence applied to (5.3) with $\eta^{\varepsilon} \rightarrow \eta$ we then obtain (5.3) in the general case. Next, by taking $\eta(\lambda)= \pm \lambda$ in (5.3) we get (5.2), thus proving that $\nu$ is an admissible mv solution.

It remains to prove the propositions. We shall estimate the $R^{i}$ using the estimates (3.3)-(3.6) and the $L_{\infty}$-estimate in Lemma 3.1. We have

$$
\begin{aligned}
\left|R^{3}\right|+\left|R^{6}\right| & \leq C h(h+\delta) \sum_{K \in T_{h}} \int_{K}|L(U)|\left\|\eta^{\prime} \phi\right\|_{W^{2, \infty}} d x d t \\
& \leq C h(h+\delta) \sum_{K \in T_{h}} \int_{K}|L(U)|\left(|\nabla U|^{2}+|\nabla U|+1\right)\|\phi\|_{W^{2, \infty}(K)} d x d t \\
& \leq C \frac{h \delta}{\bar{\delta}} \int_{S^{N}} \varepsilon_{1}(U)|\nabla U|^{2}+C h(h+\delta)\|L(U)\|_{L_{2}\left(S^{N}\right)} \\
& \leq C\left(\frac{h \delta}{\bar{\delta}}+h \sqrt{\delta}\right) .
\end{aligned}
$$

Using also Lemma 4.1, we have

$$
\begin{aligned}
\left|R^{4}\right| & \leq \sum_{n=0}^{N} \sum_{K \in T_{h}^{n}} C h^{2} \int_{K \cap R_{n}^{2}}\left|U_{+}-U_{-}\right|\left(\left|\nabla_{x} U\right|^{2}+\left|\nabla_{x} U\right|+1\right)\|\phi\|_{W^{2, \infty}(K)} d x \\
& \leq C h \int_{S^{N}}|\tilde{U}|\left|\nabla_{x} U\right|^{2} d x d t+C h^{3 / 2}\left(\sum_{n=0}^{N}\left\|U_{+}-U_{-}\right\|_{L^{2}\left(R_{n}^{2}\right)}^{2}\right)^{1 / 2} \\
& \leq C\left(\frac{h}{\overline{\bar{\delta}}}+h^{3 / 2}\right) .
\end{aligned}
$$


Further,

$$
\begin{aligned}
R^{5} & =\delta \int_{S^{N}} L(U)^{2} \eta^{\prime \prime}(U) \phi d x d t+\delta \int_{S^{N}} L(U)\left(\phi_{t}+\sum_{i} f_{i}^{\prime} \phi_{x_{i}}\right) \eta^{\prime}(U) d x d t \\
& \geq-C \delta\|L(U)\|_{L_{2}\left(S^{N}\right)} \geq-C \sqrt{\delta}
\end{aligned}
$$

To estimate $R^{7}$ and $R^{8}$, we shall use the following result.

Proposition 5.3. We have

$$
U_{t}\left(\pi\left(\eta^{\prime}(U)\right)\right)_{t} \geq 0, \quad \nabla_{x} U \cdot \nabla_{x}\left(\pi\left(\eta^{\prime}(U)\right)\right) \geq 0 .
$$

Proof. We see that $U_{t}\left(\pi\left(\eta^{\prime}(U)\right)\right)_{t}$ is constant on each tetrahedron $K \in T_{h}$. According to the construction of the tetrahedrons $K$, there is for each $K$ an orthogonal coordinate transformation in the $x_{1}, x_{2}$-plane such that $K$ always has one edge in each coordinate direction. Hence, let $\left(x_{1}, x_{2}, t\right), t_{1}^{\prime} \leq t \leq t_{2}^{\prime}$, be such an edge of $K$; then the sign of $U_{t} \pi\left(\eta^{\prime}(U)\right)_{t}$ is equal to the sign of $\left(U\left(x_{1}, x_{2}, t_{1}^{\prime}\right)-U\left(x_{1}, x_{2}, t_{2}^{\prime}\right)\right)\left(\eta^{\prime}\left(U\left(x_{1}, x_{2}, t_{1}^{\prime}\right)\right)-\eta^{\prime}\left(U\left(x_{1}, x_{2}, t_{2}^{\prime}\right)\right)\right)$, which is nonnegative since $\eta^{\prime}$ is nondecreasing. An analogous argument in the $x_{i}$ directions will then, after summation, complete the proof of Proposition 5.3, since $\nabla_{x} v \cdot \nabla_{x} w$ is invariant under orthogonal coordinate transformations.

Let $\left.\mathscr{P} \varphi\right|_{K} \in P_{0}(K) \forall K \in T_{h}$ be defined by

$$
\left.\mathscr{P} \varphi\right|_{K}=\int_{K} \varphi d x d t /\left(\int_{K} d x d t\right) \quad \forall \varphi \in L_{2}\left(R_{+}^{3}\right),
$$

i.e., $\mathscr{P} \varphi$ is the $L_{2}$-projection of $\varphi$ onto the set of piecewise constants. Then using (5.6), we have

$$
\begin{aligned}
& R^{7}= \sum_{\substack{K \in T_{h} \\
K \subset S^{N}}} \int_{K} \varepsilon_{1}(U) \nabla U \cdot \nabla\left(\pi\left(\eta^{\prime} \phi\right)-\pi\left(\eta^{\prime} \mathscr{P} \phi\right)\right) d x d t \\
&+\sum_{\substack{K \in T_{h} \\
K \subset S^{N}}} \int_{K} \varepsilon_{1}(U) \nabla U \cdot \nabla \pi\left(\eta^{\prime} \mathscr{P} \phi\right) d x d t \equiv \mathrm{I}+\mathrm{II}, \\
&|\mathrm{I}| \leq C \sum_{\substack{K \in T_{h} \\
K \subset S^{N}}} \int_{K} \varepsilon_{1}(U)\left\|\nabla\left(\eta^{\prime}(\phi-\mathscr{P} \phi)\right)\right\|_{L_{\infty}(K)}|\nabla U| d x d t \\
& \leq C \sum_{\substack{K \in T_{h} \\
K \subset S^{N}}} \int_{K} \varepsilon_{1}(U)\left(h|\nabla U|^{2}+|\nabla U|\right)\|\nabla \phi\|_{L_{\infty}(K)} d x d t \\
& \leq C h \int_{S^{N}} \varepsilon_{1}(U)|\nabla U|^{2} d x d t+C\left(\int_{S^{N}} \varepsilon_{1}(U)|\nabla U|^{2} d x d t\left\|\varepsilon_{1}(U)\right\|_{L_{\infty}\left(S^{N}\right)}\right)^{1 / 2} \\
& \leq C(h+\sqrt{\bar{\delta} / h)}
\end{aligned}
$$

where we have used that

$$
\|(\mathrm{I}-\mathscr{P}) \phi\|_{L_{\infty}(K)} \leq C h\|\nabla \phi\|_{L_{\infty}(K)}
$$

and the inverse estimate (3.6) to obtain

$$
\left\|\varepsilon_{1}(U)\right\|_{L_{\infty}\left(R_{+}^{3}\right)} \leq C \bar{\delta} / h .
$$


Next, by Proposition 5.3, and since $\mathscr{P} \phi$ is piecewise constant, we have

$$
\mathrm{II}=\sum_{\substack{K \in T_{n} \\ K \subset S^{N}}} \int_{K} \varepsilon_{1}(U) \nabla U \cdot \nabla\left(\pi\left(\eta^{\prime}\right)\right) \mathscr{P} \phi d x d t \geq 0,
$$

so that

$$
R^{7} \geq-C(h+\sqrt{\bar{\delta}} / h)
$$

Finally,

$$
\begin{aligned}
R^{8}= & \sum_{\substack{K \in T_{h} \\
K \subset S^{N}}} \int_{K} \varepsilon_{2}(U) \nabla_{x} U \cdot \nabla_{x}\left(\pi\left(\eta^{\prime} \phi\right)-\pi\left(\eta^{\prime} \mathscr{P} \phi\right)\right) d x d t \\
& +\sum_{\substack{K \in T_{n} \\
K \subset S^{N}}} \int_{K} \varepsilon_{2}(U) \nabla_{x} U \cdot \nabla_{x} \pi\left(\eta^{\prime} \mathscr{P} \phi\right) d x d t \equiv \mathrm{III}+\mathrm{IV}
\end{aligned}
$$

As above, we get by Lemma 4.1

$$
\begin{aligned}
|\mathrm{III}| & \leq C h \overline{\bar{\delta}} \sum_{n=0}^{N} \int_{R_{n}^{2}}|\tilde{U}| \nabla_{x} U \cdot \nabla_{x}\left(\pi\left(\eta^{\prime}(\phi-\mathscr{P} \phi)\right)\right) d x \\
& \leq C h \overline{\bar{\delta}} \sum_{\substack{K \in T_{n} \\
K \subset S^{N}}} \sum_{n=0}^{N} \int_{K}|\tilde{U}|\left|\nabla_{x} U\right|\left\|\nabla_{x}\left(\eta^{\prime}(\phi-\mathscr{P} \phi)\right)\right\|_{L_{\infty}\left(K \cap R_{n}^{2}\right)} d x \\
& \leq C \overline{\bar{\delta}} \sum_{K \in T_{n}} \int_{K}|\tilde{U}|\left|\nabla_{x} U\right|\left\|\nabla_{x}\left(\eta^{\prime}(\phi-\mathscr{P} \phi)\right)\right\|_{L_{\infty}(K)} d x d t \\
& \leq C \overline{\bar{\delta}} \sum_{K \subset S^{N}} \int_{K \in T_{n}}|\tilde{U}|\left(h\left|\nabla_{x} U\right|^{2}+\left|\nabla_{x} U\right|\right)\left\|\nabla_{x} \phi\right\|_{L_{\infty}(K)} d x d t \\
& \leq C h \int_{S^{N}} \varepsilon_{2}(U)\left|\nabla_{x} U\right|^{2} d x d t+C\left(\int_{S^{N}} \varepsilon_{2}(U)\left|\nabla_{x} U\right|^{2} d x d t\left\|\varepsilon_{2}(U)\right\|_{L_{\infty}}\right)^{1 / 2} \\
& \leq C(h+\sqrt{\bar{\delta}}) .
\end{aligned}
$$

Finally, by Proposition 5.3,

$$
\mathrm{IV}=\sum_{\substack{K \in T_{h} \\ K \subset S^{N}}} \int_{K} \varepsilon_{2}(U) \nabla_{x} U \cdot \nabla_{x}\left(\pi\left(\eta^{\prime}(U)\right)\right) \mathscr{P} \phi d x d t \geq 0 .
$$

Letting now $h \rightarrow 0$ in the above estimates, we obtain Proposition 5.1.

Proof of Proposition 5.2. Let us introduce the cutoff function

$$
\psi(x, t)=\left\{\begin{array}{l}
1 \quad \text { if } \beta(\tilde{R}+1-|x|)+t<0 \\
\exp (-(\beta(\tilde{R}+1-|x|)+t) / \tau) \quad \text { otherwise }
\end{array}\right.
$$

where

$$
\frac{1}{\beta}=8 \sup _{|w| \leq\left\|U_{h}^{h}\right\|_{L_{\infty}}}\left(\left|f_{1}^{\prime}(w)\right|,\left|f_{2}^{\prime}(w)\right|\right)
$$

and

$$
\tau=C(h+\delta+\overline{\bar{\delta}}+\bar{\delta} / h)
$$


where $C$ is a sufficiently large constant. We now take $M=1 / \beta$ in the definition of $V_{h}^{n}$. Further, we note that $\left.\psi\right|_{R_{+}^{3} \backslash \Omega_{\tilde{R}+1, M}}=1$ and $\psi$ is exponentially decreasing in $\Omega_{\tilde{R}+1, M}$. We then have for $U=U^{h}$ and $N=1,2,3, \ldots$ the following local stability result:

$$
\begin{aligned}
& \frac{1}{2}\left[\int_{R_{N+1}^{2}} U_{-}^{2} \psi d x+\sum_{n=0}^{N} \int_{R_{n}^{2}}\left(U_{+}-U_{-}\right)^{2} \psi d x\right] \\
& \quad+\delta \int_{S^{N}}(L(U))^{2} \psi d x d t+\int_{S^{N}} \varepsilon_{1}(U)|\nabla U|^{2} \psi d x d t \\
& \quad+\int_{S^{N}} \varepsilon_{2}(U)\left|\nabla_{x} U\right|^{2} \psi d x d t \leq C \int u_{0}^{2} \psi d x .
\end{aligned}
$$

A similar stability estimate is proven in Theorem 1.2 of [4]. Since $u_{0} \in L_{\infty}\left(R^{2}\right)$ and supp $u_{0} \subset\{x:|x| \leq \tilde{R}\}$, we obtain from (5.7)

$$
\int_{R_{N}^{2}} U_{ \pm}^{2} \psi d x \leq C \tau e^{-\beta / \tau}
$$

so that by the inverse estimate $(3.6 \mathrm{~b})$,

$$
\|U\|_{L_{\infty}\left(\left(R_{+}^{3} \backslash \Omega_{\tilde{R}+1,1 / \beta}\right) \cap\{t<T\}\right)} \leq C \frac{\sqrt{\tau}}{h} e^{-\beta / \tau}
$$

which is the desired result.

6. Proof of Lemma 3.3 (The Initial Condition). Here we prove that the $L_{1}$-stability (2.8) and the initial condition (2.9) are satisfied. First we note by the definition of $V_{h}$ that $\left\langle\nu_{(x, t)},|\lambda|\right\rangle$, i.e., the $L_{\infty}$ weak-star limit of $\left|U^{h}(x, t)\right|$, has support in $\Omega_{\tilde{R}+2, M}$ (i.e., compact support in $x$ for fixed $t$ ). Next, by the following $L_{2}$-stability,

$$
\left\|U^{h}(\cdot, t)\right\|_{L_{2}\left(R^{2}\right)} \leq \exp (C h / \delta)\left\|u_{0}\right\|_{L_{2}\left(R^{2}\right)},
$$

which is obtained from the stability estimate (3.3) by a Gronwall inequality as in (4.3), we get

$$
\left\|U^{h}(\cdot, t)\right\|_{L_{1}\left(R^{2}\right)} \leq C\left\|u_{0}\right\|_{L_{2}\left(R^{2}\right)}, \quad t<T .
$$

Now using that $g\left(U^{h}(x, t)\right) \rightarrow\left\langle\nu_{(\bar{x}, t)}, g(\lambda)\right\rangle$ in the $L_{\infty}$ weak-star topology for every continuous state variable $g$, we obtain from (6.1) and (6.2)

$$
\int_{R^{2}}\left\langle\nu_{(x, t)}, \lambda^{2}\right\rangle d x \leq \int_{R^{2}} u_{0}^{2} d x \quad \text { a.e. } t \in(0, T)
$$

and

$$
\int_{R^{2}}\left\langle\nu_{(x, t)},|\lambda|\right\rangle d x \leq C \quad \text { a.e. } t \in(0, T)
$$

which proves (2.8).

To prove (2.9), we shall use a technique (see [1]) which involves the following weak convergence and the $L_{2}$-stability (6.3):

Proposition 6.1. For $\phi \in \mathscr{C}_{0}^{1}\left(\left\{x \in R^{2}:|x|<\tilde{R}+2\right\}\right)$ we have

$$
\lim _{t \rightarrow 0} \int_{R^{2}}\left\langle\nu_{(x, t)}, \lambda\right\rangle \phi d x=\int_{R^{2}} u_{0} \phi d x \text {. }
$$


We postpone the proof of this result to the end of this section. Assuming first that $u_{0} \in \mathscr{C}_{0}^{1}\left(R^{2}\right)$ and $\operatorname{supp} u_{0} \subset\left\{x \in R^{2}:|x| \leq \tilde{R}\right\}$, we have with the aid of Proposition 6.1 and (6.3),

$$
\begin{aligned}
\underset{t \rightarrow 0}{\limsup } \int_{R^{2}}\left\langle\nu_{(x, t)},\left(\lambda-u_{0}\right)^{2}\right\rangle d x \\
\quad=\limsup _{t \rightarrow 0} \int_{R^{2}}\left\langle\nu_{(x, t)}, \lambda^{2}-u_{0}^{2}-2 u_{0}\left(\lambda-u_{0}\right)\right\rangle d x \\
\quad \leq-2 \limsup _{t \rightarrow 0} \int_{R^{2}}\left\langle\nu_{(x, t)}, \lambda-u_{0}\right\rangle u_{0} d x=0 .
\end{aligned}
$$

Further, by using that $\left\langle\nu_{(x, t)},\left|\lambda-u_{0}\right|\right\rangle$ has its support in $\Omega_{\tilde{R}+2, M}$ and Jensen's inequality, we get

$$
\begin{aligned}
& \limsup _{t \rightarrow 0} \int_{R^{2}}\left\langle\nu_{(x, t)},\left|\lambda-u_{0}(x)\right|\right\rangle d x \\
& \quad \leq C \limsup _{t \rightarrow 0}\left(\int_{R^{2}}\left\langle\nu_{(x, t)},\left(\lambda-u_{0}\right)^{2}\right\rangle d x\right)^{1 / 2}=0,
\end{aligned}
$$

which proves the initial condition (2.9) for regular initial data. In the more general case $u_{0} \in L_{\infty}\left(R^{2}\right)$ with supp $u_{0} \subset\left\{x \in R^{2}:|x| \leq \tilde{R}\right\}$, we let the functions $f_{n}$ satisfy $f_{n} \in \mathscr{C}_{0}^{1}\left(R^{2}\right), \operatorname{supp} f_{n} \subseteq\left\{x \in R^{2}:|x| \leq \tilde{R}\right\}$ and $\lim _{n \rightarrow \infty}\left\|f_{n}-u_{0}\right\|_{L_{2}\left(R^{2}\right)}=0$, and use (6.3) and Jensen's inequality to obtain

$$
\lim _{t \rightarrow 0} \int_{R^{2}}\left\langle\nu_{(x, t)},\left(\lambda-u_{0}\right)^{2}\right\rangle d x=\lim _{n \rightarrow \infty} \lim _{t \rightarrow 0} \int_{R^{2}}\left\langle\nu_{x, t},\left(\lambda-f_{n}\right)^{2}\right\rangle .
$$

Now, as in (6.4) we have

$$
\lim _{n \rightarrow \infty} \lim _{t \rightarrow 0} \int_{R^{2}}\left\langle\nu_{(x, t)},\left(\lambda-f_{n}\right)^{2}\right\rangle d x=0
$$

so that

$$
\limsup _{t \rightarrow 0} \int_{R^{2}}\left\langle\nu_{(x, t)},\left|\lambda-u_{0}\right|\right\rangle d x \leq \lim _{t \rightarrow 0} C\left(\int_{R^{2}}\left\langle\nu_{(x, t)},\left(\lambda-u_{0}\right)^{2}\right\rangle d x\right)^{1 / 2}=0 .
$$

We now turn to the proof of Proposition 6.1. Let $\phi \in \mathscr{C}_{0}^{1}\left(R^{2}\right), \operatorname{supp} \phi \subset\{x \in$ $\left.R^{2}:|x| \leq \tilde{R}\right\}, \psi \in \mathscr{C}_{0}^{1}([0, T)), \psi(0)=1$ and take $v=\pi(\phi \psi)$ in (3.1). Letting then $h$ tend to zero, we obtain as in (5.2)

$$
\begin{aligned}
& \int_{R_{+}} \int_{R^{2}}\left\langle\nu_{(x, t)}, \lambda\right\rangle \phi d x \psi_{t} d t \\
& \quad+\int_{R_{+}} \int_{R^{2}} \sum_{i}\left(\left\langle\nu_{(x, t)}, f_{i}(\lambda)\right\rangle \phi_{x_{i}}\right) d x \psi d t+\int_{R^{2}} u_{0} \phi d x=0 .
\end{aligned}
$$

Further, we define the functions $A, B \in L_{\infty}((0, T))$ by

$$
A(t)=\int_{R^{2}}\left\langle\nu_{(x, t)}, \lambda\right\rangle \phi(x) d x, \quad B(t)=\int_{R^{2}} \sum_{i}\left\langle\nu_{(x, t)}, f_{i}(\lambda)\right\rangle \phi_{x_{i}}(x) d x .
$$

Since $\nu_{y}$ is a measure-valued solution, we have $A_{t}+B=0$ in the sense of distributions on $R_{+}$. We note that $B \in L_{1}((0, T))$, which implies $A_{t} \in L_{1}(0, T)$. Hence, $A$ has bounded variation and $\lim _{t \rightarrow 0} A(t)$ exists. Taking now

$$
\psi \equiv \psi_{n}= \begin{cases}(1-n t)^{2}, & t \leq 1 / n \\ 0, & t>1 / n\end{cases}
$$


in (6.6), we get

$$
\int_{R^{2}} u_{0} \phi d x=-\lim _{n \rightarrow \infty} \int_{R^{2}} A(t)\left(\psi_{n}\right)_{t} d t=\lim _{t \rightarrow 0} A(t),
$$

which proves the proposition.

Remark 6.1. An alternative method to prove the initial condition (2.9) is to establish the strong $L_{1}$ convergence

$$
\lim _{t \rightarrow 0}\left\|U^{h}(\cdot, t)-u_{0}\right\|_{L_{1}\left(R^{2}\right)}=0
$$

by using standard error estimates with respect to a solution having smooth initial data. This is possible, since for short times such a solution remains smooth. Using this method, we get (2.9) choosing also $\delta=C h$. Recall that to obtain (6.3) we assumed $h / \delta \rightarrow 0$ as $h \rightarrow 0$, cf. Remark 6.2.

Remark 6.2. The following argument, cf. [1], proves that Theorem 3.1 holds also for the case $\delta=h$. Analogously to (6.6) and (5.3), we obtain

$$
\begin{aligned}
& \int_{R_{+}} \int_{R^{2}}\left\langle\nu_{(x, t)}, \lambda^{2}\right\rangle \phi d x \psi_{t} d t \\
& \quad+\int_{R_{+}} \int_{R^{2}} \sum_{i}\left(\left\langle\nu_{(x, t)}, \bar{q}_{i}(\lambda)\right\rangle \phi_{x_{i}}\right) d x \psi d t+\int_{R^{2}}\left(u_{0}\right)^{2} \phi d x \geq 0,
\end{aligned}
$$

where $\bar{q}_{i}$ are the entropy fluxes corresponding to the entropy $\lambda^{2}$ and $\phi, \psi$ are as in (6.6). Using now that $\nu$ satisfies (5.3) with $\eta=\lambda^{2}$ we note (cf. [1]) that the integral

$$
\bar{A}(t) \equiv \int_{R^{2}}\left\langle\nu_{(x, t)}, \lambda^{2}\right\rangle \phi(x) d x
$$

has bounded variation as a function of $t$ and hence the $\operatorname{limit}_{\lim } \lim _{t \rightarrow 0} \bar{A}(t)$ exists. Taking now $\psi$ as in (6.7) and $\phi(x)=1$ for $x \in \Omega_{\tilde{R}+2, M}$, we have by (6.8)

$$
\lim _{t \rightarrow 0} \int_{R^{2}}\left\langle\nu_{(x, t)}, \lambda^{2}\right\rangle d x \leq \int_{R^{2}}\left(u_{0}\right)^{2} \phi d x
$$

which combined with Proposition 6.1 proves (6.4) and (6.5) as above. We thus conclude that Lemma 3.3 holds also for $\delta=h$.

7. Error Estimates for the SC-Method. In this section we prove that the SC-method applied with piecewise polynomials of order $k$ to the linear problem

$$
\begin{array}{ll}
L(u) \equiv u_{t}+\sum_{i=1}^{d} a_{i} u_{x_{i}}=0 & \text { in } R^{d} \times R_{+}, \\
u(\cdot, 0)=u_{0} & \text { on } R^{d},
\end{array}
$$

with smooth solution has essentially the same order of convergence as the corresponding streamline diffusion finite element method (see [4]) obtained by choosing $\varepsilon_{1}=\varepsilon_{2}=0$ in $(3.1)$, i.e., $\mathscr{O}\left(h^{k+1 / 2}\right)$. Introducing the bilinear form

$$
\begin{aligned}
B_{M}(v, w)= & \int_{S^{M}} L(v)(w+\delta(L(w))) d x d t \\
& +\sum_{n=1}^{M} \int_{R_{n}^{d}}\left(v_{+}-v_{-}\right) w_{+} d x+\int_{R_{0}^{d}} v_{+} w_{+} d x,
\end{aligned}
$$


the SC-method for (7.1) can be formulated, now with $V_{h}$ consisting of piecewise polynomials of degree $k$ : Find $U \in V_{h}$ such that for $M=0,1,2, \ldots$

$B_{M}(U, v)+\int_{S^{M}} \varepsilon_{1}(U) \nabla U \cdot \nabla v d x d t+\int_{S^{M}} \varepsilon_{2}(U) \nabla_{x} U \cdot \nabla_{x} v d x d t=\int_{R_{0}^{d}} u_{0} v_{+} d x$ with $\varepsilon_{1}$ and $\varepsilon_{2}$ as in Section 3. For the quantity $e=U-\pi u \in V_{h}$, we have

$$
\begin{aligned}
B_{M}(e, e) & +\int_{S^{M}} \varepsilon_{1}(U)|\nabla e|^{2} d x d t+\int_{S^{M}} \varepsilon_{2}(U)\left|\nabla_{x} e\right|^{2} d x d t \\
= & B_{M}(u-\pi u, e)-\int_{S^{M}} \varepsilon_{1}(U) \nabla(\pi u) \cdot \nabla e d x d t \\
& -\int_{S^{M}} \varepsilon_{2}(U) \nabla_{x} \pi u \cdot \nabla_{x} e d x d t \\
\equiv & \mathrm{I}+\mathrm{II}+\mathrm{III} .
\end{aligned}
$$

Now

$$
\begin{aligned}
B_{M}(e, e)= & \frac{1}{2}\left(\left\|e_{-}\right\|_{L_{2}\left(R_{M+1}^{d}\right)}^{2}+\sum_{n=1}^{M}\left\|e_{+}-e_{-}\right\|_{L_{2}\left(R_{n}^{d}\right)}^{2}+\left\|e_{+}\right\|_{L_{2}\left(R_{0}^{d}\right)}^{2}\right) \\
& +\delta \int_{S^{M}}(L(e))^{2} d x d t
\end{aligned}
$$

and with the notation $\eta=u-\pi u$, we have

$$
\begin{aligned}
\mathrm{I}=B_{M}(\eta, e) \leq & \frac{1}{2} B(e, e)+\sum_{n=1}^{M+1}\|\eta\|_{L_{2}\left(R_{n}^{d}\right)}^{2} \\
& +\delta^{-1}\|\eta\|_{L_{2}\left(S^{M}\right)}^{2}+\delta\|L(\eta)\|_{L_{2}\left(S^{M}\right)}^{2} .
\end{aligned}
$$

Further, by (3.6),

$$
\begin{aligned}
\mathrm{II} \leq & \frac{\delta}{8} \int_{S^{M}}(L(U))^{2} d x d t+\frac{2(\bar{\delta})^{2}}{\delta} \int_{S^{M}}|\nabla(\pi u)|^{2}|\nabla e|^{2} d x d t \\
\leq & \frac{\delta}{4} \int_{S^{M}}(L(e))^{2} d x d t+\frac{\delta}{4} \int(L(\pi u))^{2} d x d t \\
& +C \frac{(\bar{\delta})^{2}}{\delta h^{2}}\|\nabla \pi u\|_{L_{\infty}}^{2} \int_{S^{M}} e^{2} d x d t
\end{aligned}
$$

and finally, by Lemma 4.1 ,

$$
\begin{aligned}
\mathrm{III} & \leq \frac{c}{h} \int_{S^{M}}|\tilde{U}|^{2} d x d t+C h \overline{\bar{\delta}}^{2} \int_{S^{M}}\left|\nabla_{x} \pi u\right|^{2}\left|\nabla_{x} e\right|^{2} d x d t \\
& \leq \frac{1}{8} \sum_{n=0}^{M}\left\|e_{+}-e_{-}\right\|_{L_{2}\left(R_{n}^{d}\right)}^{2}+C\left\|\nabla_{x} \pi u\right\|_{L_{\infty}}^{2} \frac{\overline{\bar{\delta}}^{2}}{h} \int_{S^{M}} e^{2} d x d t .
\end{aligned}
$$

Combining now (7.2)-(7.6) and the interpolation estimate (3.4), with $V_{h}^{n}$ now consisting of piecewise polynomials of degree $k \geq 1$, we get for $u \in H^{2}\left(S^{M}\right) \cap$ $W^{1, \infty}\left(S^{M}\right)$

$$
B_{M}(e, e) \leq C h^{2 k}\left(\delta+h^{2} / \delta\right)+C \int_{S^{M}} e^{2} d x d t
$$

Thus by a Gronwall argument, as in (4.3), we have

$$
B_{M}(e, e) \leq C h^{2 k}\left(\delta+h^{2} / \delta\right)
$$


Using now (3.4), this proves that for $M=1,2,3, \ldots$

$$
\begin{aligned}
\left\|(U-u)_{-}\right\|_{L_{2}\left(R_{M+1}^{d}\right)}^{2}+\sum_{n=1}^{M}\left\|U_{+}-U_{-}\right\|_{L_{2}\left(R_{n}^{d}\right)}^{2} \\
\quad+\left\|\left(U-u_{0}\right)_{+}\right\|_{L_{2}\left(R_{0}^{d}\right)}^{2}+\delta \int_{S^{M}}(L(U))^{2} d x d t \leq C h^{2 k}\left(\delta+h^{2} / \delta\right),
\end{aligned}
$$

which for $\delta=C h$ gives the accuracy $\mathscr{O}\left(h^{k+1 / 2}\right)$.

Remark 7.1. Following [4], we easily get local error estimates corresponding to (7.7) away from regions where the exact solution is nonsmooth.

Acknowledgment. This work was supported by the National Swedish Board for Technical Development and the Swedish Institute of Applied Mathematics.

\author{
Department of Mathematics \\ Chalmers University of Technology \\ and University of Göteborg \\ S-412 96 Göteborg, Sweden \\ E-mail: CMSAS@SEGUC21.bitnet
}

1. R. J. DiPerna, "Measure-valued solutions of conservation laws," Arch. Rational Mech. Anal., v. 88,1985 , pp. $223-270$.

2. T. J. R. HUGHES \& M. MALLET, "A new finite element formulation for computational fluid dynamics: IV. A discontinuity-capturing operator for multidimensional advective-diffusive systems," Comput. Methods Appl. Mech. Engrg., v. 58, 1986, pp. 329-336.

3. T. J. R. HUGhES, L. P. FRANCA \& M. MALLET, "VI. Convergence analysis of the generalized SUPG formulation for linear time-dependent multidimensional advective-diffusive systems," Comput. Methods Appl. Mech. Engrg., v. 63, 1987, pp. 97-112.

4. C. Johnson, U. NÄVERT \& J. PITKÄRANTA, "Finite element methods for linear hyperbolic problems," Comput. Methods Appl. Mech. Engrg., v. 45, 1984, pp. 285-312.

5. C. JOHNSON \& J. SARANEN, "Streamline diffusion methods for problems in fluid mechanics," Math. Comp., v. 47, 1986, pp. 1-18.

6. C. JOHNSON \& A. SZEPESSY, "On the convergence of a finite element method for a nonlinear hyperbolic conservation law," Math. Comp., v. 49, 1987, pp. 427-444.

7. C. JOHNSON, A. SZEPESSY \& P. HANSBO, On the Convergence of Shock-Capturing Streamline Diffusion Finite Element Methods for Hyperbolic Conservation Laws, Preprint No 1987:21, Dept. of Math., Chalmers Univ. of Technology, S-412 96 Göteborg. (To appear in Math. Comp.)

8. S. N. KRUŽKOV, "First order quasilinear equations in several independent variables," Math. USSR-Sb., v. 10, 1970, pp. 217-243.

9. A. SZEPESSY, "Measure-valued solutions of scalar conservation laws with boundary conditions," Arch. Rational Mech. Anal. (To appear.)

10. L. TARTAR, Compensated Compactness and Applications to Partial Differential Equations, in Research Notes in Mathematics, Nonlinear Analysis and Mechanics: Heriot-Watts Symposium, vol. 4 (R. J. Knops, ed.), Pitman Press, London, 1979.

11. L. TARTAR, The Compensated Compactness Method Applied to Systems of Conservation Laws, Systems of Nonlinear Partial Differential Equations (J. M. Ball, ed.), NATO ASI series, Reidel, Dordrecht, 1983, pp. 263 285. 